\title{
Constraints on dark matter powered stars from the extragalactic background light
}

\author{
Andreas Maurer ${ }^{*, a}$, Martin Raue $^{a}$, Tanja Kneiske ${ }^{a}$, Dominik Elsässer ${ }^{b}$, Peter H. \\ Hauschildt $^{c}$, and Dieter Horns ${ }^{a}$ \\ anstitut für Experimentalphysik, Universität Hamburg \\ Luruper Chaussee 149, D-22761 Hamburg, Germany \\ ${ }^{b}$ Institut für Theoretische Physik und Astrophysik \\ Am Hubland, D-97074 Würzburg, Germany \\ ${ }^{c}$ Hamburger Sternwarte \\ Gojenbergsweg 112, D-21029 Hamburg, Germany \\ E-mail: andreas.maurerephysik •uni-hamburg • de, \\ martin.raue@physik.uni-hamburg.de, \\ tanja.kneiske@physik.uni-hamburg.de, \\ elsaessereastro.uni-wuerzburg.de, yeti@hs.uni-hamburg.de, \\ dieter.hornsephysik.uni-hamburg.de
}

\begin{abstract}
Recently, it has been proposed that self-annihilating dark matter could have a significant effect on the formation and development of the first stars in the universe. In such a model, the energy released by the self-annihilating dark matter may be the main power source for this class of young stellar objects called Dark Stars. Their features (e.g. luminosity, temperature, lifetime) could differ from normal Population III stars and therefore makes them distinguishable. The contribution from Dark Stars to the extragalactic background light considering multiple initial parameters is calculated. By comparing our results with existing data and limits of the diffuse infrared background we can derive observational constraints on Dark Stars in the early universe. Future observations (e.g. with the forthcoming James Webb Space Telescope) will improve these results.
\end{abstract}

25th Texas Symposium on Relativistic Astrophysics

December 6-10, 2010

Heidelberg, Germany

\footnotetext{
* Speaker.
} 


\section{Introduction}

Astrophysical observations from the last decades hint towards a large mass contribution in the universe that is of an unknown, "dark" nature. Large sky surveys (e.g. SDSS [1] , 2dF [2]) and numerical simulations like the Millennium run [3] point towards a convincing scenario of large scale structure formation within a cold dark matter (DM) model in an accelerated expanding universe $(\Lambda \mathrm{CDM})$. A good particle candidate for the DM content of the universe is a self-annihilating WIMP (Weakly Interacting Massive Particle, see e.g. [4, 5] for a review article). These WIMPs could affect the physics of the first stars in a significant way.

The epoch of the first star formation in the universe is not yet observable with today's astronomical instruments. The circumstances and mechanisms of these processes are still topics of ongoing analysis and rely on sophisticated numerical simulations (for a review, see e.g. [6]). Recently, several studies discussed the role of WIMP dark matter on the formation of the first stars [7, 8]. Assuming that self-annihilating particles provide the dark matter content of the universe, it is obtained that this new source of energy injection into the first stars may alter their features remarkably. The additional energy injection of these self-annihilating WIMPs delay or even prevent the nuclear hydrogen burning. Two mechanisms are available to supply the star with high DM densities: adiabatic contracted DM due to the gravitational pull from the baryons that form the star (as investigated by [7]) and elastic scattering between WIMPs and baryons (see e.g. [8]). There can also be the possibility for a combination of these two mechanisms with different relative efficiencies. The exact physical process that provides the dark matter powered stars (Dark Star; DS) with "fuel" has no great impact on the results presented here as long as there is a high enough dark matter density in the center of the DS guaranteed. In both cases the resulting features of these stellar objects can be quite generic: low surface temperatures, high luminosities and possible enhanced lifetimes compared with Population III stars $[8,9]$. After the "dark phase" the star is thought to evolve as a normal zero age main sequence star.

Instead of considering the very challenging direct detection of DS (see e.g. [10]), the approach here is to search for signatures of DS in the diffuse metagalactic radiation field (MRF). The local optical to infrared part of the MRF is also known as extragalactic background light (EBL, for a review see e.g. [11]). Its main contribution originates from integrated starlight and thermal dust emissions of all cosmic epochs. This fact makes the EBL an ideal probe for the star formation history of the universe and therefore offers a unique possibility to search for emission in the early universe, e.g. investigated in [12]. There are different types of observational strategies for the EBL. Lower limits to the EBL are derived from galaxy number counts which are available up to a redshift $\approx 2$ from the Hubble Space Telescope [13] and the Spitzer instrument [14]. Upper limits can be derived from direct observations (COBE [15]), but these are presumably polluted by prominent foreground emission. A different method for obtaining upper limits on the EBL density makes use of the spectra from very high energy (VHE) $\gamma$-ray sources, in particular blazars (see e.g. [16]). These limits deliver the possibility to constrain DS scenarios.

For all calculations here a flat Friedmann cosmology is adopted with $\Omega_{m}=0.3, \Omega_{\Lambda}=0.7$ and a Hubble constant of $H_{0}=70 \mathrm{~km} \mathrm{~s}^{-1} \mathrm{Mpc}^{-1}$. 


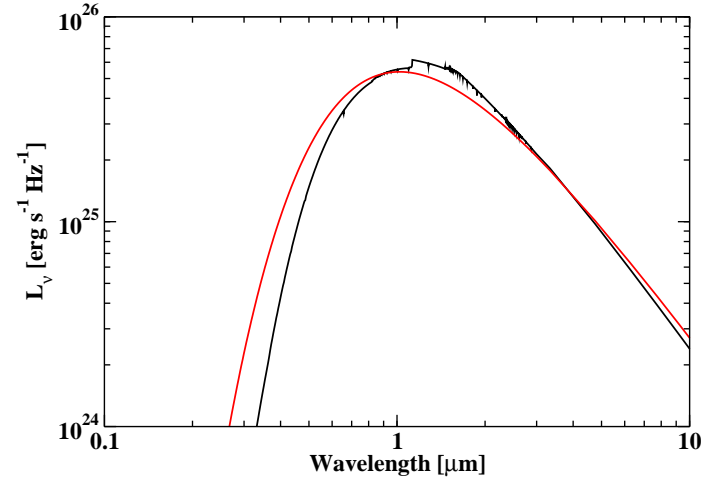

(a) Specific luminosity for a modelled DS atmosphere (black) and a blackbody (red) with same temperature and radius. DS parameters are $R_{\mathrm{DS}}=2.4 \times 10^{12} \mathrm{~m}$, $M_{\mathrm{DS}}=106 \mathrm{M}_{\odot}, T_{\mathrm{DS}}=5000 \mathrm{~K}$

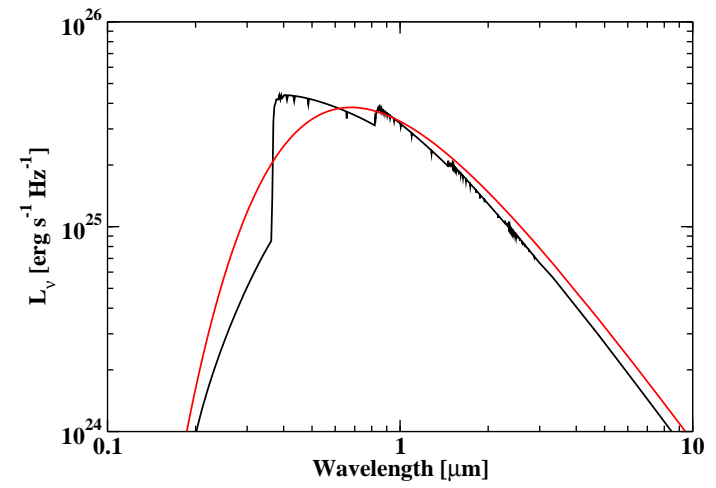

(b) Same as in (a). DS parameters are $R_{\mathrm{DS}}=1.1 \times$ $10^{12} \mathrm{~m}, M_{\mathrm{DS}}=690 \mathrm{M}_{\odot}, T_{\mathrm{DS}}=7500 \mathrm{~K}$

Figure 1: Model spectra used as input parameters. DS properties adopted from [9].

\section{Method}

To calculate the contribution of dark matter powered stars to the extragalactic background light a forward evolution model is used, see e.g. [17]. In the following the individual input parameters of the model are described.

The spectra of DS are calculated with the model atmosphere package PHOENIX, version 16 [18]. For the model atmospheres used here the abundance of $\mathrm{H}$ was set to 0.92 by number (mass fraction: 0.75 ) and that of He was set to 0.08 by number (mass fraction: 0.25 ) for all models, all other elements (including Li) have an abundance of zero in these models. DS spectra with effective temperatures $5000 \mathrm{~K}$ and $7500 \mathrm{~K}$ and with parameters adopted from [9] have been computed (see Fig. 1).

Independent of the exact mechanism powering the DM burning, models predict a stable phase in which the total radiative output of the DS is dominated by dark matter burning [7, 8]. During this phase the luminosity is nearly constant (see e.g. Figure 2 in [9], Figure 4 in [8] and Figure 1 in [19]). The exact length of the DS phase ( $\left.\Delta t_{\mathrm{DS}}\right)$ is highly uncertain and depends on various factors, e.g. DM type, DS model, DM halo profile, etc (for an extensive discussion see [10]). In this model a wide band of possible DS lifetimes is explored ranging from $10^{5}$ to $10^{9}$ years.

A crucial parameter, to which the EBL flux is sensitive, is the formation density of DS. This quantity is directly linked to the cosmic star formation rate (SFR) $\dot{\rho}_{*}(z)$ for the first stars (Pop III) which can be expressed as a comoving mass formation rate in units of $\mathrm{M}_{\odot} \mathrm{year}^{-1} \mathrm{Mpc}^{-3}$. For the model calculations here, a constant SFR for Dark Stars is assumed, ranging from the onset $\left(z_{\max }\right)$ to the end $\left(z_{\min }\right)$ of the formation epoch. The linear scaling factor $\mathrm{SFR}_{\mathrm{Norm}}$ ranges from $10^{-7}$ to $10^{-3}$ comparable to typical values obtained for the first stars [20].

The influence of $z_{\max }$ on the resulting EBL is - due to the redshift dilution - negligible and so in the following its value is set to 30 . The investigated range of $z_{\min }$ reaches from 5 to 15 which is in good agreement to simulated Population III star formation periods [21, 22]. 


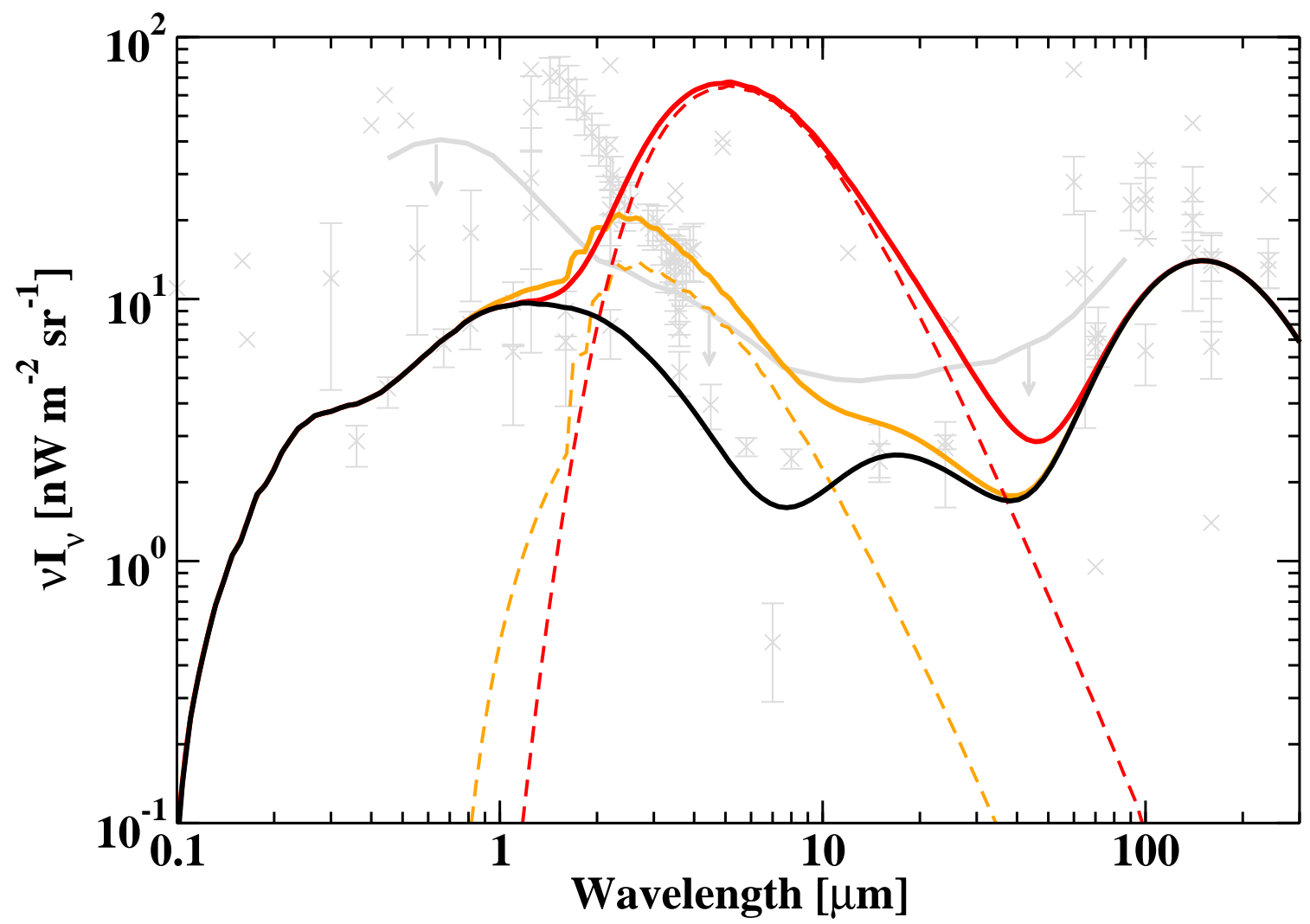

Figure 2: EBL contribution for two different Dark Star parameter sets (orange-dashed: $T_{\mathrm{DS}}=7500 \mathrm{~K}$, $M=690 \mathrm{M}_{\odot}$; red-dashed: $T_{\mathrm{DS}}=5000 \mathrm{~K}, M=106 \mathrm{M}_{\odot}$ ). Both models are calculated with $\mathrm{SFR}_{\mathrm{Norm}}=10^{-3}$, $\Delta t_{\mathrm{DS}}=10^{9}$ years, $z_{\mathrm{min}}=5$. Grey markers: EBL measurements \& limits adopted from [16]; grey: upper limits from $\mathrm{TeV}$ observations (realistic model) from [16]. Black line: EBL lower limit by [23]. The total EBL shape in presence of a DS contribution is the sum of the lower limit and the specific DS signature (orange and red lines).

\begin{tabular}{cccc}
\hline \hline & $\Delta t_{\mathrm{DS}}$ & $z_{\min }$ & $\mathrm{SFR}_{\text {Norm }}$ \\
\hline $\min$ & $10^{5}$ & 15 & $10^{-7}$ \\
fiducial & $10^{7}$ & 10 & $10^{-5}$ \\
$\max$ & $10^{9}$ & 5 & $10^{-3}$ \\
\hline
\end{tabular}

Table 1: Dark Star parameter range

\section{Results \& Conclusion}

The resulting contribution to the extragalactic background light is calculated for different sets of parameters. Both spectra of Dark Stars presented in Fig. 1 are taken as model input and a wide range of possible signatures in the infrared background are calculated.

In Fig. 2 the EBL contribution for two DS parameter sets is displayed in comparison with a strict lower limit for the guaranteed astrophysical EBL from stars and dust in galaxies [23] and upper limits from $\mathrm{TeV}$ observations [16]. All input parameters are set to the maximal values as displayed in table 1. Comparing the resulting DS EBL signatures (orange dashed and red dashed 
curves) with the their respective input spectra (see Fig. 1) it can be seen that the spectral features are smoothed by the redshift integration. The peak of the EBL contribution for both models is located at wavelengths $>2 \mu \mathrm{m}$ which differs from the value $(\lambda \sim 1 \mu \mathrm{m})$ for Pop III stars [24] as expected due to lower effective temperatures of DS. The peak of both EBL signatures clearly reaches into the detectable region of the infrared background. This shows that the EBL offers the potential to constrain DS parameter space. A DS contribution from these models would result into a total EBL which is the sum of the lower limit (black shape) and the DS signature (orange and red shapes). These EBL shapes are already disfavored by upper limits from $\mathrm{TeV}$ observations (grey line).

Further work on the constraints delivered by contributions from dark matter powered stars to the diffuse infrared background will be published in [25]. Future efforts to measure the extragalactic background light via deep galaxy counts (e.g. with the James Webb Space Telescope) and to derive refined upper limits (e.g. measurements of VHE $\gamma$-ray sources with forthcoming Cherenkov telescopes like CTA) will improve the possibility to put constraints on Dark Stars enormously.

\section{Acknowledgments}

The participation in the 25th Texas Symposium on Relativistic Astrophysics was made possible with the support of the Cluster of Excellence: Connecting Particles with the Cosmos of the Landesexzellenzinitiative Hamburg. This work has made use of NASA's Astrophysics Data System Service.

\section{References}

[1] K. N. Abazajian, J. K. Adelman-McCarthy, M. A. Agüeros, S. S. Allam, C. Allende Prieto, D. An, K. S. J. Anderson, S. F. Anderson, J. Annis, N. A. Bahcall, and et al., The Seventh Data Release of the Sloan Digital Sky Survey, Astrophys. J., Suppl. Ser. 182 (June, 2009) 543-558, [0812. 0649 ].

[2] M. Colless, B. A. Peterson, C. Jackson, J. A. Peacock, S. Cole, P. Norberg, I. K. Baldry, C. M. Baugh, J. Bland-Hawthorn, T. Bridges, R. Cannon, C. Collins, W. Couch, N. Cross, G. Dalton, R. De Propris, S. P. Driver, G. Efstathiou, R. S. Ellis, C. S. Frenk, K. Glazebrook, O. Lahav, I. Lewis, S. Lumsden, S. Maddox, D. Madgwick, W. Sutherland, and K. Taylor, The 2dF Galaxy Redshift Survey: Final Data Release, ArXiv Astrophysics e-prints (June, 2003) [astro-ph/0306581].

[3] V. Springel, S. D. M. White, A. Jenkins, C. S. Frenk, N. Yoshida, L. Gao, J. Navarro, R. Thacker, D. Croton, J. Helly, J. A. Peacock, S. Cole, P. Thomas, H. Couchman, A. Evrard, J. Colberg, and F. Pearce, Simulations of the formation, evolution and clustering of galaxies and quasars, Nature 435 (June, 2005) 629-636, [astro-ph/0504097].

[4] G. Jungman, M. Kamionkowski, and K. Griest, Supersymmetric dark matter, Phys. Rep. 267 (Mar., 1996) 195-373, [hep-ph/9506380].

[5] G. Bertone, D. Hooper, and J. Silk, Particle dark matter: evidence, candidates and constraints, Phys. Rep. 405 (Jan., 2005) 279-390, [hep-ph / 0404175$].$

[6] V. Bromm and R. B. Larson, The First Stars, Ann. Rev. Astron. Astrophys. 42 (Sept., 2004) 79-118, [astro-ph/0311019].

[7] D. Spolyar, K. Freese, and P. Gondolo, Dark Matter and the First Stars: A New Phase of Stellar Evolution, Physical Review Letters 100 (Feb., 2008) 051101-+, [astro-ph/0 05.0521 ]. 
[8] F. Iocco, A. Bressan, E. Ripamonti, R. Schneider, A. Ferrara, and P. Marigo, Dark matter annihilation effects on the first stars, Mon. Not. R. Astron. Soc. 390 (Nov., 2008) 1655-1669, [0 805.4016 ].

[9] D. Spolyar, P. Bodenheimer, K. Freese, and P. Gondolo, Dark Stars: A New Look at the First Stars in the Universe, Astrophys. J. 705 (Nov., 2009) 1031-1042, [0903.3070].

[10] E. Zackrisson, P. Scott, C. Rydberg, F. Iocco, B. Edvardsson, G. Östlin, S. Sivertsson, A. Zitrin, T. Broadhurst, and P. Gondolo, Finding High-redshift Dark Stars with the James Webb Space Telescope, Astrophys. J. 717 (July, 2010) 257-267, [1002.3368].

[11] M. G. Hauser and E. Dwek, The Cosmic Infrared Background: Measurements and Implications, Ann. Rev. Astron. Astrophys. 39 (2001) 249-307, [astro-ph / 0105539 ].

[12] M. Raue, T. Kneiske, and D. Mazin, First stars and the extragalactic background light: how recent $\gamma$-ray observations constrain the early universe, Astron. Astrophys. 498 (Apr., 2009) 25-35, [0806.2574].

[13] P. Madau and L. Pozzetti, Deep galaxy counts, extragalactic background light and the stellar baryon budget, Mon. Not. R. Astron. Soc. 312 (Feb., 2000) L9-L15, [astro-ph/9907315].

[14] G. G. Fazio, M. L. N. Ashby, P. Barmby, J. L. Hora, J.-S. Huang, M. A. Pahre, Z. Wang, S. P. Willner, R. G. Arendt, S. H. Moseley, M. Brodwin, P. Eisenhardt, D. Stern, E. V. Tollestrup, and E. L. Wright, Number Counts at $3 \mu m<\lambda<10 \mu m$ from the Spitzer Space Telescope, Astrophys. J., Suppl. Ser. 154 (Sept., 2004) 39-43, [astro-ph/ 0405595 ].

[15] M. G. Hauser, R. G. Arendt, T. Kelsall, E. Dwek, N. Odegard, J. L. Weiland, H. T. Freudenreich, W. T. Reach, R. F. Silverberg, S. H. Moseley, Y. C. Pei, P. Lubin, J. C. Mather, R. A. Shafer, G. F. Smoot, R. Weiss, D. T. Wilkinson, and E. L. Wright, The COBE Diffuse Infrared Background Experiment Search for the Cosmic Infrared Background. I. Limits and Detections, Astrophys. J. 508 (Nov., 1998) 25-43, [astro-ph/9806167].

[16] D. Mazin and M. Raue, New limits on the density of the extragalactic background light in the optical to the far infrared from the spectra of all known TeV blazars, Astron. Astrophys. 471 (Aug., 2007) 439-452, [astro-ph/0701694].

[17] T. M. Kneiske, K. Mannheim, and D. H. Hartmann, Implications of cosmological gamma-ray absorption. I. Evolution of the metagalactic radiation field, Astron. Astrophys. 386 (Apr., 2002) 1-11, [astro-ph/0202104].

[18] P. H. Hauschildt and E. Baron, A 3D radiative transfer framework. VI. PHOENIX/3D example applications, Astron. Astrophys. 509 (Jan., 2010) A36+, [0911.3285].

[19] S. Yoon, F. Iocco, and S. Akiyama, Evolution of the First Stars with Dark Matter Burning, Astrophys. J., Lett. 688 (Nov., 2008) L1-L4, [0806.2662].

[20] M. Trenti and M. Stiavelli, Formation Rates of Population III Stars and Chemical Enrichment of Halos during the Reionization Era, Astrophys. J. 694 (Apr., 2009) 879-892, [0901.0711].

[21] M. Trenti, M. Stiavelli, and J. Michael Shull, Metal-free Gas Supply at the Edge of Reionization: Late-epoch Population III Star Formation, Astrophys. J. 700 (Aug., 2009) 1672-1679, [0 905.4504 ].

[22] U. Maio, B. Ciardi, K. Dolag, L. Tornatore, and S. Khochfar, The transition from population III to population II-I star formation, Mon. Not. R. Astron. Soc. (June, 2010) 905-+, [1 003.4992 ].

[23] T. M. Kneiske and H. Dole, A lower-limit flux for the extragalactic background light, Astron. Astrophys. 515 (June, 2010) A19+, [1001.2132]. 
[24] M. Santos, V. Bromm, and M. Kamionkowski, The contribution of the first stars to the cosmic infrared background, Mon. Not. R. Astron. Soc. 336 (2002) 1082.

[25] A. Maurer, M. Raue, T. M. Kneiske, D. Elsässer, P. H. Hauschildt, and D. Horns, submitted, . 